\title{
Culture numérique et citoyenneté mondiale : quels enjeux pour l'École?
}

\section{Sylvain Genevois}

\section{(2) OpenEdition}

1 Journals

Édition électronique

URL : http://journals.openedition.org/trema/3036

DOI : 10.4000/trema.3036

ISSN : 2107-0997

Éditeur

Faculté d'Éducation de l'université de Montpellier

\section{Édition imprimée}

Date de publication : 1 décembre 2013

Pagination : $16-27$

ISSN : 1167-315X

\section{Référence électronique}

Sylvain Genevois, «Culture numérique et citoyenneté mondiale : quels enjeux pour l'École ? », Tréma [En ligne], 40 | 2013, mis en ligne le 01 décembre 2015, consulté le 01 mai 2019. URL : http:// journals.openedition.org/trema/3036 ; DOI : 10.4000/trema.3036

Ce document a été généré automatiquement le 1 mai 2019.

Trema 


\section{Culture numérique et citoyenneté} mondiale: quels enjeux pour l'École?

Sylvain Genevois

I. Introduction 
1 L'École n'a pas seulement pour mission de construire des savoirs et des savoir-faire, elle participe à la formation du futur citoyen. Dans un contexte de mondialisation, les finalités civiques et culturelles de l'École se trouvent réinterrogées, en lien avec le déclin des états-nations et les médiacultures de masse qui se mettent en place à l'échelle

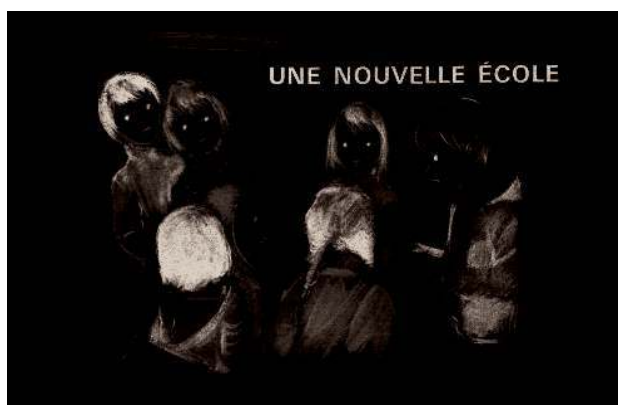
mondiale (Jacquinot, 2010). Les technologies numériques occupent une place prépondérante au sein de ces nouvelles pratiques culturelles et sociales, particulièrement pour les adolescents qui ont un usage intensif d'Internet et des réseaux sociaux. Différents auteurs mettent en avant l'intérêt d'une éducation au numérique et à la citoyenneté mondiale, en lien avec les formes d'échanges et le nouveau rapport à soi et aux autres construit par les adolescents (Tabary-Bolka, 2009; Gaudelli, 2009 ; Myers, 2010, Bennett et al. 2010). Ces compétences numériques dépassent largement la maîtrise technique des outils et interrogent directement la manière de former les jeunes au nouveau modèle de citoyen qui est en train de naître sous nos yeux. Pour Benkler (2009), les technologies de l'information et de la communication débouchent sur une culture plus critique et introspective où les citoyens ont un plus grand pouvoir par leur capacité à publier leurs propres opinions sur une série d'enjeux. En même temps qu'elle uniformise les cultures et les pratiques sociales, la mondialisation conduit, à travers la société mondiale de l'information qu'elle met en place, à construire une citoyenneté plus active, plus sociale et tournée vers le "vivre ensemble». La question est de savoir comment l'École peut accompagner l'émergence de cette nouvelle société civile et les formes de citoyenneté qui se développent à travers les réseaux numériques.

2 À travers une recherche exploratoire prenant appui sur une revue de la littérature ainsi que sur les discours des acteurs et les instructions officielles des programmes, nous proposons d'interroger les liens entre culture numérique et citoyenneté mondiale, afin d'en dégager les principaux enjeux pour l'École.

\section{De l'éducation au numérique à l'éducation à la citoyenneté mondiale : quels liens et quels débats ?}

\section{1. La culture des jeunes à l'ère de la mondialisation numérique}

3 Le développement de la culture numérique est un phénomène saillant particulièrement chez les jeunes. Il convient de préciser d'emblée: par jeunes, nous entendons ici les enfants et les adolescents. Concernant la culture numérique, nous nous référons à la définition donnée par Proulx (2002) qui désigne ainsi un ensemble de valeurs, de connaissances et de pratiques qui impliquent l'usage d'outils informatisés, mais aussi des pratiques de consommation médiatique et culturelle, de communication et d'expression de soi. En France, 96\% des jeunes de 8 à 17 ans utilisent Internet, 48\% sont connectés à un réseau social, principalement Facebook utilisé par 85\% des lycéens, 57\% des collégiens et 11\% des élèves de primaire (Étude tns sofres, 2011). Les jeunes se connectent souvent seuls, depuis leur ordinateur personnel (50\%) et leur mobile (23\%). Ces chiffres viennent 
confirmer une autre enquête sur les pratiques culturelles des Français à l'ère numérique (Donnat, 2009). Celle-ci montre que la tranche des 15-24 ans consacre en moyenne $21 \mathrm{~h}$ par semaine sur les nouveaux écrans (ordinateur ou console de jeux). Phénomène nouveau: les jeunes passent plus de temps devant ces nouveaux écrans que devant la télévision. Leurs pratiques numériques sont orientées vers les industries du divertissement (jeux vidéo, films, musiques...), mais concernent aussi la recherche d'information et les échanges sur des forums ou sur des blogs. Pour l'essentiel cette culture numérique se construit hors des murs de l'école et en dehors du contrôle des adultes (parents ou enseignants). Elle est susceptible de s'accompagner de nouvelles formes de violence et de cyberharcèlement qui peuvent prendre des conséquences dramatiques pour certains adolescents (Blaya, 2012). Mais comme le montre Lardellier (2006), ces pratiques numériques des adolescents débouchent aussi sur une nouvelle façon d'être, à la fois plus centrée sur l'individu (mise en scène du moi) et plus tournée vers les autres (culture réticulaire). Elle crée un nouveau rapport au monde pour les «natifs numériques » qui pensent et agissent différemment des générations antérieures, $\mathrm{du}$ fait d'une exposition précoce aux technologies de l'information et de la communication (Prensky, 2001). Les adolescents développent ainsi un nouveau style cognitif caractérisé par l'apprentissage multi-tâches, une attention relativement courte pendant l'apprentissage et une stratégie pour apprendre qui s'appuie sur l'exploration et la découverte.

Différents auteurs (voir par exemple Bennett et al., 2008; Baron, Bruillard, 2008) ont nuancé fortement la thèse de Prensky sur les « digital natives ». Les pratiques numériques ne sont pas le fait seulement de la nouvelle "génération Internet", mais concernent l'ensemble des citoyens confrontés aux changements culturels et sociétaux induits par la révolution numérique. Les jeunes n'ont pas un rapport inné à l'ordinateur et à Internet. Ils ont besoin d'être formés à la maitrise de l'information et de s'approprier les compétences clés pour appréhender les mediacultures qui se sont développées dans le sillage de la société mondiale de l'information. Comme le rappellent Baron et Bruillard (2008, p. 9), « les digital natives sont pour une bonne part aussi des novices, des digital naïves, des proies faciles pour les diverses incitations du marché. Leurs utilisations des technologies sont fréquentes, mais dans un spectre très limité et avec un degré d'autonomie relatif.» La culture numérique des jeunes n'est pas unique, mais plurielle. Elle varie en fonction de leurs pratiques culturelles et de leur degré de familiarisation avec les TIC.

5 Face à ces inégalités, l'éducation aux cultures numériques ${ }^{1}$ (Le Deuff, 2009) constitue un impératif pour l'École. Cette éducation ne se limite pas à une simple prise en main des outils et des services numériques. Elle doit être envisagée plus généralement comme l'appropriation d'une nouvelle culture numérique, donc comme une forme d'acculturation: elle suppose une réflexion globale sur les conditions nécessaires pour faciliter le passage entre culture numérique et culture scolaire (Cerisier, 2012). Entre ces deux cultures, le fossé semble important, voire infranchissable. Les points communs entre culture numérique et culture scolaire sont pourtant plus nombreux qu'on pourrait le penser en termes d'offre et de partage de savoirs, de développement de la coopération, de capacité d'innovation à travers de nouveaux outils (Durpaire, 2008). Cette éducation au numérique passe par une éducation aux médias, concernant notamment le traitement et la validation de l'information, la préservation de l'identité numérique, la responsabilité dans la publication de contenus numériques. Mais l'éducation aux médias ne suffit pas 
pour traiter des questions de gouvernance, de pluralisme, de diversité culturelle, de solidarité et pour comprendre plus généralement les recompositions de la citoyenneté à l'heure de la mondialisation.

\section{2. Éducation civique, éducation à la citoyenneté mondiale et « e- citoyenneté »}

6 Pour Audigier (interviewé par Pagoni, 2009, p 153), "nous sommes passés d'une citoyenneté d'appartenance et d'obéissance qui mettait en avant l'identité collective, la participation aux procédures électives et le respect des lois, à une conception de la citoyenneté qui privilégie l'affirmation de la liberté de chacun, qui se conjugue avec la mobilité des appartenances et la labilité des choix.». Cette nouvelle citoyenneté se reconfigure autour d'une constellation d'affiliations en fonction du sexe, de l'âge, de la classe sociale ou de l'appartenance ethnique. L'émergence d'une citoyenneté multiappartenance et multi-identitaire est favorisée par le développement des nouveaux medias, principalement des réseaux sociaux sur Internet. Selon Bourgeault (2005), l'éducation civique dispensée dans un cadre national en référence à des valeurs patriotiques (ce qu'il désigne comme une forme de citoyenneté de conformisme) est en train de laisser place progressivement à des formes plus larges d'éducation à la citoyenneté, à la responsabilité et à la solidarité à l'échelle mondiale.

7 Les débats sont assez forts, notamment dans la littérature anglo-saxonne, autour du concept de citoyenneté globale, de ses contours et de la place à lui accorder dans le curriculum (Buckingham, 2008, Dalhgren, 2009, Sherrod et al., 2010, Gaudelli, 2009). Nous n'avons pas la place ici de les présenter dans le détail, mais pour l'essentiel ces débats renvoient à diverses conceptions de la citoyenneté mondiale en référence à différentes aspirations : aux droits de l'homme universels, à la lutte contre les inégalités à l'échelle mondiale, à de nouvelles formes de gouvernance et de participation citoyenne à l'échelle mondiale. Face à ces différentes finalités, l'éducation à une citoyenneté mondiale ne fait pas l'unanimité. Elle se heurte surtout aux finalités civiques et patrimoniales de savoirs scolaires enseignés dans le cadre de systèmes éducatifs nationaux. Elle repose sur l'enseignement de valeurs dans un monde incertain. Elle ne remplace pas la citoyenneté nationale justifiée par des fondements historiques et conservant des liens fort avec l'appartenance à un État. Elle n'est donc pas facile à intégrer dans le curriculum formel. Le contexte évolue cependant avec la construction progressive d'une citoyenneté européenne, obligeant à dépasser les clivages nationaux traditionnels (Feyfant, 2010). Pour Osler (2010), «l'enseignement de l'histoire doit être reformulé, de manière à reconnaître que les élèves ne sont pas seulement les citoyens d'un État-nation, mais sont aussi des citoyens cosmopolites émergents qui vivent dans l'ère de la mondialisation et des droits de l'homme universels. » Cette cosmo-citoyenneté, telle que pensée par Habermas, entretient des liens étroits avec l'explosion des usages communicationnels et les nouvelles formes de partage et de mutualisation sur Internet (Endrizzi, 2012).

Pour Mathias (2009), « l'Internet citoyen n'est pas une rêverie de nanti post-industriel et il est permis de concevoir que les réseaux tissent un espace légitime d'expression de la citoyenneté. L'«e-citoyenneté » peut ainsi être métaphoriquement décrite comme résultant d'une espèce de rayonnement des réflexions sociales et politiques qui traversent effectivement les réseaux, mais aussi de l'activisme dans lequel s'engage si diversement l'ensemble polymorphe de leurs usagers. » En même temps qu'elle consacre 
le pouvoir des mass media sur l'opinion publique et qu'elle concentre les pouvoirs de domination économique et culturelle, la société mondiale de l'information accroît la puissance d'action des citoyens en capacité d'agir et de s'organiser sur les réseaux. Pour Dagnaud (2011), il s'agit de nouvelles formes d'actions et de nouveaux modes du « vivre ensemble ». La conscience d'un destin commun du genre humain se développe sur les bases matérielles d'une société info-numérique mondiale qui change les processus de composition des identités individuelles et collectives et façonne un nouveau type de « citoyenneté numérique », ce que Mathias (2005) définit comme une "e-citoyenneté ». Même si cette citoyenneté numérique mondiale n'a pas encore un sens précis pour la plupart des gens, elle s'affirme de plus en plus comme une citoyenneté réaliste et vécue (Galichet, 1998), appuyée sur des pratiques numériques quotidiennes et faisant référence à des appartenances, à des valeurs et à des comportements. Dans un monde sans véritable gouvernance globale, les citoyens exercent une forme de citoyenneté post-politique dont les fondements sont davantage culturels et sociaux. Comme le montre Myers (2010), cette ouverture à des communautés hétérogènes et à une citoyenneté globale peut rendre les adolescents imperméables à une instruction civique nationale, leur donnant une image étroite de la société.

9 Cette prise en compte du rôle des nouvelles technologies dans l'action citoyenne des jeunes est assez récente (Haste, 2009). En France, elle commence à transparaître, mais elle reste encore relativement absente dans les instructions officielles et dans les discours des acteurs.

\section{Quelles compétences numériques développer à l'École à l'ère de la société mondiale de l'information? Analyse des instructions officielles et des discours des acteurs.}

Précisons qu'en France il existe peu de textes officiels qui permettraient de relier directement l'acquisition de compétences numériques à la construction d'une éducation globale du citoyen. Nous analyserons d'abord les compétences mises en avant par les instructions officielles, puis nous nous intéresserons aux discours qui commencent à mettre l'accent sur ce type d'éducation à l'échelle européenne et mondiale.

\section{1. Une approche française centrée sur les usages du numérique et sur la certification de compétences en vue d'attester une maîtrise transversale des TIC}

11 Face aux critiques suscitées par l'enseignement de la programmation lors du plan Informatique pour Tous, la France a abandonné dès la fin des années 1980 l'enseignement de l'informatique à l'école et a mis l'accent sur les usages des technologies numériques appréhendées comme des outils au service de l'enseignement-apprentissage. En 2000, le Ministère de l'Éducation nationale met en place le Brevet informatique et Internet (B2i) dans l'enseignement primaire et secondaire. En 2005, il instaure le Certificat Informatique et Internet niveau 2 pour les enseignants (C2i2e), devenu obligatoire pour exercer le métier d'enseignant à partir de 2010. Les compétences proposées par le B2i et le C2i concernent principalement l'appropriation de l'environnement informatique de travail, 
l'adoption d'une attitude responsable, la création et le traitement de données, la recherche et la maîtrise de l'information, la communication et l'échange avec les outils numériques. Ces deux référentiels de certification ont été conçus à la fin des années 1990 et au début des années 2000, ce qui explique qu'ils prennent peu en compte des compétences jugées indispensables à l'ère des réseaux sociaux. Il faut attendre 2011 pour voir une nouvelle version du B2i qui commence à intégrer les compétences du web social (web 2.0), telle par exemple la protection des données personnelles sur la Toile. Mais pour l'essentiel il s'agit de mettre l'accent sur les dangers d'Internet et assez peu de développer une culture informationnelle reposant sur une culture politique et civique (Devauchelle et al., 2010, Le Deuff, 2009). C'est en ce sens que des sites éducatifs de prévention comme Internet responsable ou Internet sans craintes ont été mis à disposition du public scolaire pour accompagner les jeunes dans la maîtrise des usages d'Internet ${ }^{2}$. Pour autant cette nouvelle version du B2i correspond davantage à un toilettage qu'à une véritable prise en compte d'un Internet citoyen. Il ne met guère en valeur les formes d'engagement citoyen, les projets collaboratifs ou les formes de solidarité permises par la Toile.

12 Une ambiguïté fondamentale apparait dans la visée de ces certifications B2i et C2i : il s'agit avant tout d'acquérir des compétences professionnelles et assez secondairement de former le citoyen de demain. Comme le souligne Hirtt (2008), le terme de compétences explose littéralement à partir des années 1990 et 2000, au moment précis où l'on commence à parler de "société de la connaissance», de «mondialisation» et de « globalisation ». Il s'agit, selon Nico Hirtt, de favoriser l'employabilité des futurs formés auxquels l'école doit garantir un socle minimum de compétences pour trouver un emploi. Depuis 2006, l'attestation de compétences numériques ne relève d'ailleurs plus du certificat B2i, mais du "Socle commun de connaissances et de compétences", indispensable à valider au cours de la scolarité obligatoire. Ces compétences numériques sont rassemblées dans le pilier 4 du socle commun qui met l'accent sur la double finalité critique et professionnelle des TIC: "la culture numérique implique l'usage sûr et critique des techniques de la société de l'information. Il s'agit de l'informatique, du multimédia et de l'internet, qui désormais irriguent tous les domaines économiques et sociaux ». Ces compétences numériques font partie des compétences transversales, mais de fait elles ne sont pas véritablement reliées aux compétences sociales et civiques qui relèvent du pilier 6: «il s'agit aussi de développer le sentiment d'appartenance à son pays, à l'Union européenne, dans le respect dû à la diversité des choix de chacun et de ses opinions politiques ». Entre ces deux piliers, le lien logique n'est ni facile ni évident à établir.

13 Si on observe par ailleurs les programmes d'éducation civique ou d'ECJS, on constate qu'ils ne font guère allusion aux enjeux de la mondialisation et des technologies numériques. Alors qu'ils consacrent une large place à la connaissance des institutions françaises et aux droits et devoirs individuels, les programmes d'éducation civique de collège-lycée n'attachent guère d'importance aux nouvelles composantes de la citoyenneté mondiale, si ce n'est l'allusion assez générale dans le programme de classe de Cinquième à "la diversité et aux identités multiples de la personne». On peut citer cependant l'exception du nouveau programme de Troisième (2012) qui accorde une place aux spécificités d'Internet dans la fabrication de l'opinion publique (Partie II sur « la vie démocratique ", thème 3 sur "l'opinion publique et les médias »). Mais le thème est présenté du point de vue de l'éducation aux médias, finalement assez peu sous l'angle d'une citoyenneté mondiale numérique à construire. De même le nouvel enseignement 
« Droit et enjeux du monde contemporain », introduit depuis 2012 en Terminale L, met en avant des thèmes concernant la "gouvernance mondiale » ou le problème du respect du "droit sur Internet», mais dans une approche qui privilégie l'initiation aux questions juridiques plus que la formation du citoyen. En ECJS, le thème de la mondialisation qui permettait d'étudier les formes d'action mondiale du citoyen a disparu du nouveau programme de Terminale paru en 2012. Pour ainsi dire, il n'existe pas véritablement d'éducation à la citoyenneté numérique dans le curriculum français. Celle-ci est quasiment absente des programmes d'éducation civique ainsi que de la formation aux TIC. C'est en ce sens que le ministre de l'Éducation nationale Vincent Peillon a annoncé son souhait de "faire entrer l'École dans l'ère du numérique» dans le but d'«accompagner une transformation majeure de civilisation», en particulier "pour préparer nos enfants à la société qui est la leur et dans laquelle ils doivent être des citoyens " : une ambition légitime qui ne semble cependant pas dénuée d'intérêt national dans la mesure où il s'agit de "défendre notre culture et nos valeurs sur les nouveaux territoires numériques $»^{3}$.

\section{2. Vers une approche plus large de l'éducation au numérique à l'échelle européenne et mondiale}

14 À l'échelle de l'Union européenne, on observe la même approche par compétences qu'en France. C'est en soi logique puisque le Socle commun de compétences est une déclinaison du Cadre de Référence Européen de 2006 concernant les «Compétences clés pour l'éducation et la formation tout au long de la vie. » On y retrouve exactement la même séparation entre les compétences numériques (pilier 4) et les compétences sociales et civiques (pilier 6). En revanche, la réflexion est plus large dans la mesure où les instances européennes ont pris conscience de la nécessité d'ancrer la citoyenneté dans un cadre supranational : « alors que la mondialisation continue à poser de nouveaux défis à l'Union européenne, chaque citoyen devra avoir à son actif un large éventail de compétences clés pour s'adapter avec souplesse à un monde évoluant rapidement et caractérisé par un degré d'interconnexion élevé »4. L'échelle mondiale semble désormais indispensable à prendre en compte dans toute éducation citoyenne. Le centre Nord-Sud du Conseil de l'Europe développe une approche plus volontariste en évoquant explicitement la nécessité d'une "éducation à la citoyenneté mondiale ». Celle-ci vise, selon le Conseil de l'Europe, à « faire prendre conscience et à renforcer la capacité citoyenne à entreprendre des actions, à défendre ses droits et à s'engager dans les débats politiques concernant la justice sociale et le développement durable aux niveaux local, national et international. Elle encourage les élèves et les enseignants à collaborer sur des problèmes globaux et permet aux citoyens de comprendre les réalités et autres processus complexes du monde d'aujourd'hui tout en développant des valeurs, des attitudes, des connaissances et des capacités qui leur donneront les moyens de relever les défis d'un monde interconnecté. $»^{5}$ Même si on en reste à un niveau assez général, le lien entre mondialisation numérique et citoyenneté mondiale est ici clairement établi. Le Conseil de l'Europe publie d'ailleurs un Guide pratique sur l'éducation à la citoyenneté mondiale, où l'éducation aux médias dans la société numérique figure à la fois comme un moyen et comme un objectif (Carvalho da Silva Miguel, 2008).

15 En Suisse romande, l'Éducation à la citoyenneté mondiale (ECM) fait partie du plan d'études depuis 2011. Elle s'articule avec l'éducation au développement durable (EDD) et 
avec la multiculturalité. Elle s'appuie sur un guide pédagogique complet mis à disposition des enseignants et des élèves, dans lequel la mondialisation est envisagée comme une question socialement vive qui concerne tous les citoyens invités à appréhender les enjeux et à prendre position. La dimension «citoyenneté numérique » est cependant limitée dans ce guide qui ne mentionne que "l'usage critique d'Internet». Au Québec, un véritable Programme de citoyenneté numérique ( $\mathrm{PCN}$ ) a été défini par la commission scolaire Lester-B.-Pearson ${ }^{6}$. Mais elle ne concerne que les écoles primaires et secondaires de l'Ouest de l'île de Montréal dans un contexte d'approche multiculturel. Il faut se tourner vers les pays anglo-saxons pour trouver une réflexion plus aboutie sur l'éducation à la citoyenneté mondiale à l'ère du numérique (Gaudelli, 2009, Haste, 2009). Mais cela ne signifie pas pour autant qu'elle soit déjà intégrée dans les curricula.

\section{Les défis d'une éducation à la citoyenneté numérique mondiale}

\section{1. De l'Internet citoyen à la construction d'une " e-citoyenneté »}

Comme nous l'avons souligné, en France les efforts se limitent essentiellement aux droits fondamentaux de l'individu et à la défense d'usages responsables sur Internet. C'est en ce sens que le code de l'éducation a été modifié pour s'adapter à la loi n 2011-302 du 22 mars 2011 portant diverses dispositions d'adaptation de la législation au droit de l'Union européenne en matière de santé, de travail et de communications électroniques. Le code de l'éducation (article L. 312-15) prévoit désormais : «dans le cadre de l'enseignement d'éducation civique, les élèves sont formés afin de développer une attitude critique et réfléchie vis-à-vis de l'information disponible et d'acquérir un comportement responsable dans l'utilisation des outils interactifs, lors de leur usage des services de communication au public en ligne. Ils sont informés des moyens de maîtriser leur image publique, des dangers de l'exposition de soi et d'autrui, des droits d'opposition, de suppression, d'accès et de rectification prévus par la loi nº 78-17 du 6 janvier 1978 relative à l'informatique, aux fichiers et aux libertés, ainsi que des missions de la Commission nationale de l'informatique et des libertés. ». D'après ce texte réglementaire, on en reste à la défense d'un Internet citoyen et non véritablement à la construction progressive d'une « e-citoyenneté ».

Nous voyons cependant émerger peu à peu la volonté de mobiliser l'École dans la construction d'une citoyenneté numérique "positive" accompagnant les évolutions sociales et culturelles actuelles. Un rapport d'information de l'Assemblée nationale concernant « les droits de l'individu dans la révolution numérique » (Bloche, Verchère, 2011) semble témoigner d'un début d'évolution vers une éducation à la citoyenneté numérique. La première partie du rapport porte assez classiquement sur l'accès à l'information et à la culture, la liberté d'expression, le respect de la vie privée, les problèmes soulevés par la numérisation du patrimoine ou la copie numérique. En revanche les deuxième et troisième parties abordent plus spécifiquement Internet comme "nouvel instrument au service de la démocratie » et comme source "de nouveaux rapports entre les citoyens et l'État». Dans ce contexte, le rôle de l'École est mis en avant : " pour accompagner les élèves dans leur appropriation de la société numérique, l'éducation nationale doit former les citoyens numériques de demain, en transmettant les valeurs civiques dans la société de l'information.» Parmi les orientations, le rapport 
propose de « mettre en place un enseignement spécifique d'éducation au numérique dans le cadre de l'éducation civique et de réfléchir à son articulation avec le B2i » (Orientation $\left.\mathrm{n}^{\circ} 36, \mathrm{p} 223\right)$. Et le rapport de conclure : « c'est en s'adressant au “citoyen numérique”, en lui permettant d'user de sa raison et de sa liberté, que l'on pourra faire de l'univers numérique un lieu d'épanouissement des droits des individus ».

On en reste à des principes généraux qui ne disent cependant pas précisément quels pourraient être les défis à relever pour établir cette «citoyenneté numérique ». Nous proposons pour finir d'identifier quelques défis importants pour l'École.

\section{2. Quelques propositions pour établir une éducation à la citoyenneté numérique}

19 La citoyenneté numérique ne se limite pas à la maîtrise des TIC, elle renvoie à un ensemble de manières de faire, de penser et d'agir dans le monde d'aujourd'hui. C'est plutôt une façon de comprendre les défis auxquels font face tous les utilisateurs des technologies numériques. À partir des lacunes rencontrées dans les textes officiels que nous avons présentés, il semble possible de formuler quelques propositions pour établir une éducation à la citoyenneté numérique :

- Élargir l'éducation civique à une véritable Éducation à la citoyenneté mondiale (ECM). Au sein d'un système éducatif qui a déjà multiplié les formes d'» éducation à » (éducation au développement durable, éducation aux médias, éducation à la santé, éducation à la sécurité...), il semble peu réaliste d'ajouter une » éducation au numérique » qui existe déjà, mais se limite le plus souvent à une prise en main des outils. En revanche, il semble souhaitable d'élargir l'éducation à la citoyenneté à une éducation à la citoyenneté numérique prenant en compte les transformations de la citoyenneté à l'heure d'Internet (communication ouverte, partage de connaissances, possibilités d'action citoyenne à l'échelle mondiale...).

- Orienter l'éducation au numérique vers une éducation socialement plus inclusive. L'objectif est de permettre d'exercer sa liberté d'expression dans sa dimension " active » à travers la messagerie électronique, les réseaux sociaux, les blogs et autres forums de discussion. C'est en effet une caractéristique majeure d'Internet d'offrir à l'internaute la capacité de contribuer à la diffusion de l'information et de participer à la circulation et à l'échange d'idées et d'opinions. Il s'agit d'identifier des possibilités d'action pour le citoyen et non seulement de sensibiliser aux règles juridiques d'Internet, aussi importantes soient ces règles par ailleurs.

- Renforcer la « dimension citoyenne » du B2i et du C2i, afin de mieux articuler ces référentiels de compétences avec l'éducation aux médias qui reste de fait peu prise en charge sur le terrain. Il existe encore manifestement un fossé important entre le discours affiché et la situation réelle en ce qui concerne l'appréhension des médiacultures.

\section{Conclusion}

$\mathrm{Au}$ terme de cette recherche exploratoire, nous voudrions souligner un paradoxe: le développement massif de la culture numérique crée une citoyenneté mondiale de fait qui n'est pas prise en compte dans les prescriptions. La mise en place progressive d'une éducation à la citoyenneté mondiale commence à s'effectuer dans certains pays 
européens, mais reste encore très limitée en France. À l'heure d'Internet et de la mondialisation, la plupart des discours officiels sur les enjeux du numérique à l'École mettent l'accent sur la nécessité d'acquérir des compétences clés dans le domaine des technologies numériques afin de maîtriser leurs usages dans la vie privée, sociale ou professionnelle. Cette éducation au numérique vise à développer des "usages sûrs et critiques» (usages responsables d'Internet), mais rarement à susciter une réflexion sur l'avènement d'une citoyenneté numérique mondiale ("e-citoyenneté »). Sans doute l'École est-elle imprégnée d'une certaine vision néolibérale où les technologies sont censées développer d'abord l'accès à l'emploi et assez secondairement l'esprit critique. Or il semble important aujourd'hui, au moment où l'expression citoyenne se développe massivement sur Internet, d'intégrer dans l'éducation civique un versant éducation à la citoyenneté numérique. Cela suppose de ne pas en rester à une approche identitaire nationale et d'identifier les nouvelles possibilités d'action du citoyen à l'ère de la mondialisation numérique.

\section{BIBLIOGRAPHIE}

Baron (Georges-Louis), Bruillard (Éric), « Technologies de l'information et de la communication et indigènes numériques : quelle situation? », Revue STICEF, Volume 15, 2008.

Bennett (Sue), Maton (Karl), Kervin (Lisa), « The digital natives debate : a critical review of the evidence », British Journal of Educational Technology, Volume 39, p. 775-786, 2008.

Bennett (W. Lance), Freelon (Deen), Wells (Chris), « Changing Citizen Identity and the Rise of a Participatory Media Culture », in Sherrod (Lonnie R.), Torney-Purta (Judith), Flanagan, (Constance A.), Handbook of Research on Civic Engagement in Youth, Wiley, p 161-188, 2010.

Benkler (Yochai), La Richesse des réseaux (trad. Anna Clercq-Roques), Lyon : Presses universitaires de Lyon, 2009.

Blaya (Catherine). Les ados dans le cyberespace. Prises de risque et cyberviolence. Coll. Pédagogie en développement, De Boeck, 2012.

Bloche (Patrick), Verchère (Patrice), Rapport d'information sur les droits de l'individu dans la révolution numérique, Assemblée nationale, juin 2011. http://www.assemblee-nationale.fr/13/rapinfo/i3560.asp

Bourgeault (Gérard). Quelle éducation ? Pour quelle cité ? Éléments de réflexion pour un débat sur l'éducation à la citoyenneté, in Duhamel (André), Jutras (France) (dir.), Enseigner et éduquer à la citoyenneté. Québec : Les Presses de l'Université Laval. p. 135-149, 2005.

Buckingham (David), Youth, Identity and Digital Media, Massachusetts Institute of Technology, 2008.

Carvalho da Silva Miguel (dir.), Guide pratique sur l'éducation à la citoyenneté mondiale. Lisbonne : Centre Nord-Sud du Conseil de l'Europe, 2008.

Cerisier (Jean-François), « La culture numérique dans le champ de l'éducation, quelques références bibliographiques », Distances et médiations des savoirs, 2012. http://dms.revues.org/163

Dagnaud (Monique), Génération Y : Les jeunes et les réseaux sociaux, de la dérision à la subversion.

Paris : Presses de Sciences Po, 2011. 
Dalhgren (Peter), Media and civic cosmopolitanism : emergence of a perspective, Conférence à

l'Université Sorbonne Nouvelle dans le cadre de l'ASSIC, 2009.

Devauchelle (Bruno), Platteaux (Hervé), Cerisier (Jean-Fançois), « Culture informationnelle, culture numérique, tensions et relations. Le cas des référentiels C2i niveau 2 », Les Cahiers du numérique, 2009/3 Vol. 5, p. 51-69.

Donnat (Olivier), Les pratiques culturelles des Français à l'ère numérique. Enquête 2008, Paris : La découverte /Ministère de la Culture et de la Communication, 2009.

Durpaire (Jean-Louis), Les jeunes, la culture numérique et le savoir scolaire, intervention effectuée à l'IUFM de Tours (mai 2008). http://documentation.tice.ac-orleanstours.fr/php5/images/stories/ pdf/conferencejldurpaire130508.pdf

Endrizzi (Laure), « Jeunesses 2.0 : les pratiques relationnelles au cœur des médias sociaux ». Dossier d'actualité Veille et Analyses, n71, février 2012, http://ife.ens-lyon.fr/vst/DA-Veille/71fevrier-2012.pdf

Enquête TNS sofres, L'usage des réseaux sociaux chez les 8-17 ans, étude réalisée en auprès de 1200 jeunes de 8-17 ans pour l'UNAF, Action Innocence et la CNIL juin 2011 http://www.tnssofres.com/etudes-et-points-de-vue/lusage-des-reseaux-sociaux-chez-les-8-17-ans

Feyfant (Annie), «L'éducation à la citoyenneté ». Dossier d'actualité de la VST, n57, octobre, 2010. http://ife.ens-lyon.fr/vst/DA/ListeDossiers.php?/57-octobre-2010.php

Galichet (François), L'éducation à la citoyenneté, Paris : Anthropos, 1998.

Gaudelli (William), Heuristics of Global Citizenship Discourses towards Curriculum Enhancement, Journal of curriculum theorizing, 2009, 25-1, p. 68-85.

Haste (Helen), « What is 'competence' and how should education incorporate new technology's tools to generate "competent civic agents », Curriculum Journal, 20 (3), p. 207-223, 2009.

Hirtt (Nico), Seront-ils des citoyens critiques? Bruxelles : Aped, 2008.

Jacquinot-Delaunay (Geneviève), De l'éducation aux médias aux médiacultures, faire évoluer théories et pratiques, INA SUP, 2011. http://www.ina-expert.com/e-dossier-de-l-audiovisuel-qu-enseigne-limage-qu-enseigner-par-l-image/de-l-education-aux-medias-aux-mediacultures-faire-evoluertheories-et pratiques.html

Mathias (Paul), « Pour un concept de l'"e-citoyenneté"». Revue internationale Sens public, 2005. http://www.sens-public.org/IMG/pdf/SensPublic_PMathias_ecitoyennete.pdf

Myers (John), « To benefit the world by whatever means possible : Adolescents constructed meanings for global citizenship », British Educational Research Journal, vol. 36, n 3, p. 483-502, 2010.

Lardellier (Pascal), Le pouce et la souris : enquête sur la culture numérique des ados, Paris : Fayard, 2006.

Le Deuff (Olivier), « Penser la conception citoyenne de la culture de l'information », Les Cahiers du numérique, 2009/3 Vol. 5, p. 39-49.

Mathias (Paul), Pour un concept de l'"e-citoyenneté", 2005. http://www.sens-public.org/spip.php? article129

Osler (Audrey), « Patriotism, multiculturalism and belonging : Political discourse and the teaching of history ", Educational review, vol. 61, n 1, p. 85-100, 2009 
Pagoni (Maria), «Rencontre avec François Audigier : Éducation à la citoyenneté et participation ", Carrefours de l'éducation, 2009/2 n² 28, p. 150-156.

Proulx (Serge), «Les formes d'appropriation d'une culture numérique comme enjeu d'une société de savoir ». Annales des télécommunications, n³-4, p. 180-189, 2002.

Prensky (Mark), « Digital natives, Digital immigrants », On the Horizon, 9 (5), 2001.

Sherrod (Lonnie R.), Torney-Purta (Judith), Flanagan (Constance A.), « Citizenship Education : A Critical Look at a Contested Field », Handbook of Research on Civic Engagement in Youth, p 161-188, 2010.

Tabary-Bolka (Laure), « Culture adolescente vs culture informationnelle ». L'adolescent acteur de la circulation de l'information sur internet, Les Cahiers du numérique, 2009/3 Vol. 5, p. 85-97.

\section{NOTES}

1. Pour LE DEUFF (2009), l'expression « cultures numériques» (au pluriel) renvoie à différentes formes de cultures chez les adolescents (du simple utilisateur au hacker par exemple) et permet de sortir du prisme de la culture unique et légitime. Selon cet auteur, l'éducation aux cultures numériques ne se réduit pas à la maîtrise de la culture informatique, mais comprend aussi l'éducation aux médias et à la culture de l'information au sens large.

2. Portail «Internet responsable» $d u$ Ministère de l'Éducation nationale: http:// eduscol.education.fr/internet-responsable/. Site «Internet sans craintes» soutenu par la Commission européenne : http://www.internetsanscrainte.fr/

3. "Faire entrer l'École dans l'ère du numérique " (discours de Vincent Peillon, 13 décembre 2012) : http://eduscol.education.fr/cid66564/faire-entrer-l-ecole-dans-l-ere-du-numerique.html

4. Recommandation du Parlement européen et du Conseil du 18 décembre 2006 sur les compétences clés pour l'éducation et la formation tout au long de la vie (p 13).

5. Centre Nord-Sud du Conseil de l'Europe http://www.coe.int/t/dg4/nscentre/default_FR.asp

6. Programme de citoyenneté numérique : http://dcp.lbpsb.qc.ca/about_fr.html

\section{RÉSUMÉS}

À l'heure d'Internet et de la mondialisation, la plupart des discours officiels sur les enjeux du numérique à l'École mettent l'accent sur la nécessité d'acquérir des compétences clés dans le domaine des technologies numériques afin de maîtriser leurs usages dans la vie privée, sociale ou professionnelle. Cette éducation au numérique vise essentiellement à développer des « usages sûrs et critiques » (cadre européen de compétences publié en 2006), mais rarement à susciter une réflexion sur l'avènement d'une citoyenneté (numérique) mondiale. À travers une recherche empirique prenant appui sur les discours des acteurs et sur les instructions officielles des programmes, nous proposons d'interroger les liens entre culture numérique et citoyenneté mondiale, afin d'en dégager les principaux enjeux pour l'École.

At the time of the Internet and globalization, most of the official discourses on digital issues at School emphasize the need to acquire key competencies in the field of digital technologies to 
control their use in private, social or professional life. This digital education is essential to develop "safe and critical practices" (European framework for key competencies published in 2006), but rarely to stimulate reflection on the emergence of a world (digital) citizenship. Through an empirical research on the actors' discourses and official curriculum prescriptions, we propose to examine the relationship between digital literacy and global citizenship, in order to identify key issues for the school.

INDEX

Mots-clés : citoyenneté mondiale, culture numérique, éducation globale, mondialisation Keywords : digital culture, global citizenship, global education., globalization

\section{AUTEUR}

\section{SYLVAIN GENEVOIS}

Maître de conférences en sciences de l'éducation et TICE. Laboratoire École, Mutations, Apprentissages - EA 4507. Université de Cergy-Pontoise 